\section{Einleitung: Infektionsmanagement und Lebensstandard}

\author{
N. Suttorp ${ }^{1}$ \\ ${ }^{1}$ Med. Klinik-Infektiologie und Pneumologie, Charité, \\ Universitätsmedizin Berlin
}

1860 erreichten in Liverpool 35\% der Menschen das 40. Lebensjahr. 60\% aller Todesfälle zur damaligen Zeit in England waren Folge von Infektionskrankheiten. Im Jahre 2000 erreichten 50\% der Menschen in England das 80. Lebensjahr [1]. Der substanzielle Anstieg der Lebenserwartung ist im Wesentlichen das Ergebnis von drei sehr wirksamen Maßnahmen: Hygiene, Vakzine und Antiinfektiva. Wenn wir an diesen drei Fronten schwächeln, hat das gravierende Folgen für unseren Lebensstandard bis hin zu einer Reduktion der Lebenserwartung.

Bei der Einhaltung der Hygiene-Regeln können wir noch besser werden. Weitere Impfstoffe sind notwendig, und große Probleme gibt es wegen der Resistenzproblematik bei den Antiinfektiva, insbesondere bei den Antibiotika.

Im ersten Teil dieser Veranstaltung starten wir daher mit aktuellen Entwicklungen bei den Antibiotikaresistenzen.
Neben Hygiene, Vakzine und Antiinfektiva wollen wir im Verlauf der Veranstaltung auch adjunktive Therapie bei schweren Infektionen als weiteres Maßnahmeninstrument beleuchten.

Danksagung: Eigene Arbeiten wurden durch das BMBF (CAPNETZ, PROGRESS, CAPSyS) sowie durch die DFG (SFB-TR84) unterstützt.

Interessenkonflikte: Es bestehen keine Interessenkonflikte.

\section{Literatur}

1 Casanova J-L, Abel $L$. Inborn errors of immunity to infection: the rule rather than exception. J Exp Med 2005: 197-201

\section{Bibliografie}

DOI http://dx.doi.org/10.1055/s-0033-1358025

Drug Res 2014; 64, Suppl. 1: S2-S2

(c) Georg Thieme Verlag KG Stuttgart · New York .

ISSN 2194-9379

\section{Korrespondenzadresse}

Norbert Suttorp

Charité - Campus Mitte

Charitéplatz 1

10117 Berlin

norbert.suttorp@charite.de 